\title{
RELATIONSHIP BETWEEN FOOD CONTACT TIME TO THE EFFECT ON TRANSFER OF MICROBES FROM CERAMIC FLOOR USING THE FIVE-SECOND RULE
}

\section{Taufan Adityawardhana*1, Agung Dwi Wahyu Widodo ${ }^{2}$, Nancy Margaritta Rehatta ${ }^{3}$}

${ }^{1}$ Medical Education Research and Staff Development Unit, Faculty of Medicine, Universitas Airlangga, Indonesia ${ }^{2}$ Department of Microbiology, Faculty of Medicine, Universitas Airlangga, Indonesia ${ }^{3}$ Department of Anesthesiology and Reanimation, Medical Education Research and Staff Development Unit, Faculty of Medicine, Universitas Airlangga, Indonesia

ABSTRACT
Eosin-Methylene Blue (EMB) in order to isolate gram-negative
bacteria (Escherichia coli) as the contamination level indicator.
Microbes identified with gram staining and observed under a light
microscope. The result reported into 5 categories: microbes were
founded or not in the petri dishes, determine whether lactose
fermentation/acid production can be observed, grade the
microbes concentration founded in the petri dishes (grade 1-6),
classify the level of contamination (low-high), describing colonies
shape in EMB agar and identifying the microbes with gram
staining. The results was at the student center's canteen 3 of 5
samples under 5 seconds are positive and 5 of 7 samples until 300
seconds are positive. The level of contamination was inconsistent
with increasing time. Whereas in the diagnostic center's canteen
12 of 12 samples were all positives, regardless of time. In
conclusion, the five-second rule is a significant oversimplification
of what actually happens when bacteria transfer from a surface to
food. Risk of transfer of contamination is constantly present
regardless of time.

\section{ARTICLE HISTORY}

Received: November 5, 2020

Revised: June 28, 2021

Accepted: June 28, 2021

Published: June 30, 2021

(Online)

doi:

10.20473/jcmphr.v2i1.26469

\section{KEYWORDS}

Five-second rule, microbes transfer, food contamination, contact time

\section{CORRESPONDING AUTHOR \\ Taufan Adityawardhana $\triangle$ taufanaditya@yahoo.com Medical Education Research and Staff Development Unit, Faculty of Medicine, Universitas Airlangga, Surabaya, Indonesia}

\begin{abstract}
INTRODUCTION
The five-second rule is a popular belief that stated food fallen to the ground needs at least 5 seconds for the microbes to transfer from the ground to the food, so it is safe to eat if it is picked up within the time. ${ }^{1}$ In Indonesia it is famous with belum lima menit, which changes the five-second rule into five-minute rule.
\end{abstract}

If the food is contaminated and continues to be eaten, gastroenterological disease such as diarrhea will be most possible to happen. Bacterial that can cause diarrhea primarily transferred via contaminated food or water. ${ }^{2}$ In a condition where Indonesia's environment was already not clean enough and the diarrhea itself is still a major health problem in Indonesia, ${ }^{3}$ there is still a possible risk factor for people believing the five-second-rule. The five-second rule is about the contact time of the fallen food to the floor, where if it is under 5 seconds the food said will not be contaminated or not contaminated enough to cause a disease. Previous study stated microbes able to survive and still able to contaminate foods from dry surface even after long duration. ${ }^{1}$ 
Therefore understanding the value of the contact time between the food and the floor surface will prove whether the five-second rule could be applied or not.

\section{MATERIALS AND METHODS}

These are 2 experimental studies at diagnostic center's canteen and study center's canteen where 12 food samples dropped to the floor using a time from 1 second until 300 seconds or 5 minutes. 5 samples for the first 5 seconds and 7 samples until 300 seconds. Food samples cultured at Eosin-Methylene Blue (EMB) agar to isolate gram-negative bacteria, especially Escherichia coli, as the contamination level indicator. Microbes identified with gram staining and observed under light microscope.

This is a descriptive experimental study. Research will be done at two places in Surabaya : diagnostic center's canteen and study center's canteen. Cheese cubes with $1 \mathrm{~cm} \times 1 \mathrm{~cm} \times 1 \mathrm{~cm}$ in size were prepared as a food sample, the size determined to fit into the test tube. There are 12 food samples dropped to the floor using a time from 1 second until 300 seconds or 5 minutes, 5 samples for the first 5 seconds (1 second, 2 seconds, 3 seconds, 4 seconds, 5 seconds) and 7 samples until 300 seconds (10 seconds, 30 seconds, 60 seconds, 120 seconds, 180 seconds, 240 seconds and 300 seconds or 5 minutes). 1 control negative used as the quality control, control negative sample will not be dropped to the floor at all but put directly to the test tube containing nutrient broth. The difference of perception between Indonesian and western people about the 5 minutes or 5 seconds were considered to determine the contact time needed. Food samples dropped with exact same amount of heights and after fall food samples not allowed to make any contact other than to the ceramic floor (ex. Shoes, walls), this controls the dropped food. Food samples placed directly from the floor into test tubes containing nutrient broth using sterilized pinset. Test tubes containing food samples being incubated in $37^{\circ} \mathrm{C}$ for 24 hours.

Incubated samples taken from a test tube using sterile ose to be streaked in a petri dish containing Eosin Methylene Blue (EMB) agar. Selective media of EMB agar were chosen to specifically locate Escherichia coli and gram negative microbes, which are a sign of floor contamination. Then the petri dishes being incubated again in $37^{\circ} \mathrm{C}$ for 24 hours.

Results from the petri dishes will be observed and be described. Sample also will be taken to undergo gram-staining method to be identified under the light microscope, whether it is gram negative or gram positive microbes. The result of experiment were classified into 5 categories : microbes were founded or not in the petri dishes, determine whether lactose fermentation/acid production can be observed, grade the microbes concentration founded in the petri dishes, classify the level of contamination, describing colonies shape in EMB agar and identifying the microbes until the level of gram staining.

The grade of contamination will be based on how much the colonies formed and rank them from the fewest to the most contaminated (0-6). After the rankings, their contamination level will be classified into low (grade 0-2), moderate (grade 3-5), 
or high (grade 6). The researcher made itself the grading and classification system based on the references. ${ }^{4,5}$ After all culture has been shown data will be compiled. Results will be analyzed descriptively and concluded based on observation of microbial culture in the petri dish.

\section{RESULTS}

Researcher was just able to distinguish the grade from 0-6. If perfect coloni shape found the contamination level should be low because by the first sterak colonies were already founded, it is later to be ranked based on how much are the colonies and the shape of colonies to observe the possibility of two or more different species based on distinguished colonies characteristics. If colonies are immeasurable but the streaking able to be observed the contamination level will be moderate. High levels of contamination will just be observed when Escherichia coli is most likely to exist, which green metallic color observed in the petri dish. The results of samples from the student center's canteen floor could be seen as examples (Figure 1$3)$.

Table 1. Relationship between Food Contact Time to The Effect on Transfer of Microbes in Student Center's Canteen Floor

\begin{tabular}{ccccc}
\hline $\begin{array}{c}\text { Student Center's } \\
\text { Canteen }\end{array}$ & $\begin{array}{c}\text { Detection of Microbial } \\
\text { Growth in EMB Agar }\end{array}$ & $\begin{array}{c}\text { Lactose } \\
\text { Fermentation }\end{array}$ & $\begin{array}{c}\text { Grade of Microbes } \\
\text { Concentration } \\
\text { (0-6) }\end{array}$ & $\begin{array}{c}\text { Rank of } \\
\text { Contamination Level }\end{array}$ \\
\hline 1 second & No & No & 0 & Low \\
2 seconds & Yes & No & 1 & Low \\
3 seconds & No & No & 0 & Low \\
4 seconds & Yes & No & 5 & Moderate \\
5 seconds & Yes & No & 2 & Low \\
10 seconds & No & Yes & 3 & Low \\
30 seconds & Yes & Yes & 6 & High \\
60 seconds & Yes & No & 2 & Low \\
120 seconds & Yes & No & 0 & Low \\
180 seconds & No & No & 2 & Low \\
240 seconds & Yes & Yes & 4 & Moderate \\
300 seconds & Yes & No & 0 & Low \\
Control negative & No & & & . \\
\hline
\end{tabular}

Table 2. Relationship between Food Contact Time to The Effect on Transfer of Microbes in Diagnostic Center's Canteen Floor

\begin{tabular}{ccccc}
\hline $\begin{array}{c}\text { Diagnostic Center's } \\
\text { Canteen }\end{array}$ & $\begin{array}{c}\text { Detection of Microbial } \\
\text { Growth in EMB Agar }\end{array}$ & $\begin{array}{c}\text { Lactose } \\
\text { Fermentation }\end{array}$ & $\begin{array}{c}\text { Grade of Microbes } \\
\text { Concentration } \\
\text { (0-6) }\end{array}$ & $\begin{array}{c}\text { Rank of } \\
\text { Contamination Level }\end{array}$ \\
\hline 1 second & Yes & Yes & 6 & High \\
2 seconds & Yes & Yes & 6 & High \\
3 seconds & Yes & Yes & 6 & High \\
4 seconds & Yes & Yes & 6 & High \\
5 seconds & Yes & Yes & 6 & High \\
10 seconds & Yes & Yes & 6 & High \\
30 seconds & Yes & Yes & 6 & High \\
60 seconds & Yes & Yes & 6 & High \\
120 seconds & Yes & Yes & 6 & High \\
180 seconds & Yes & Yes & 6 & High \\
240 seconds & Yes & No & 0 & High \\
300 seconds & Yes & Low & \\
Control negative & & & 6 & 6 \\
\hline
\end{tabular}


(A)
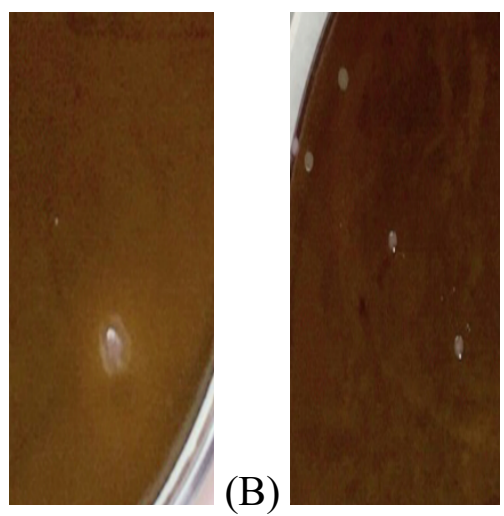

(C)
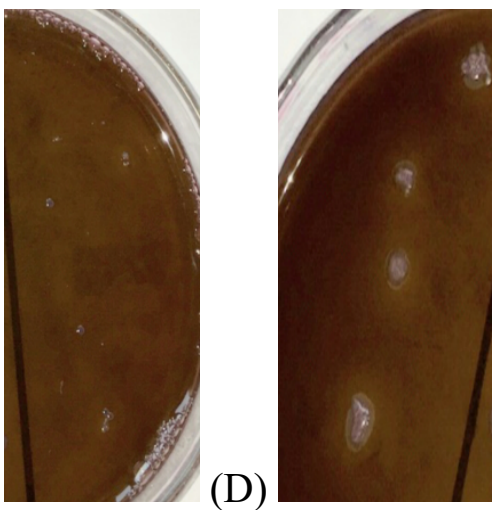

Figure 1. The Results of Low Level Contamination. (A) Grade 1 in "2 Seconds" Specimen; (B) Grade 2 in " 5 Seconds" Specimen; (C) Grade 2 in "120 Seconds" Specimen; (D) Grade 2 in"240 Seconds" Specimen

(A)

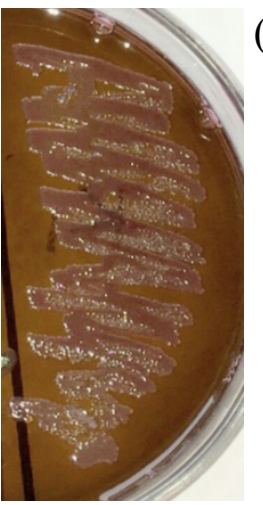

(B)

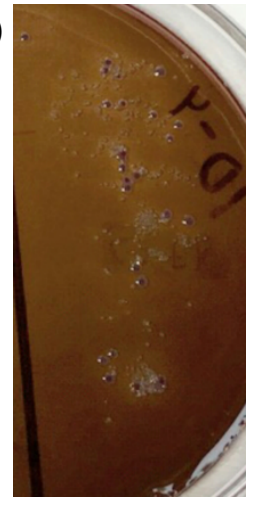

(C)

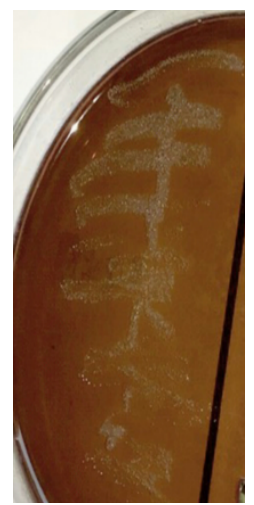

Figure 2. The Results of Moderate Level Contamination. (A) Grade 5 in"4 Seconds" Specimen; (B) Grade 3 in "30 Seconds" Specimen; (C) Grade 4 in "300 Seconds" Specimen

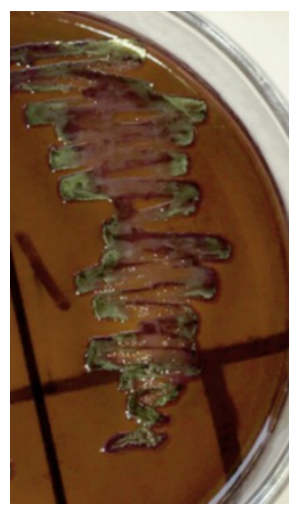

Figure 3. The Results of High Level Contamination with Grade 6 in "60 Seconds" Specimen 


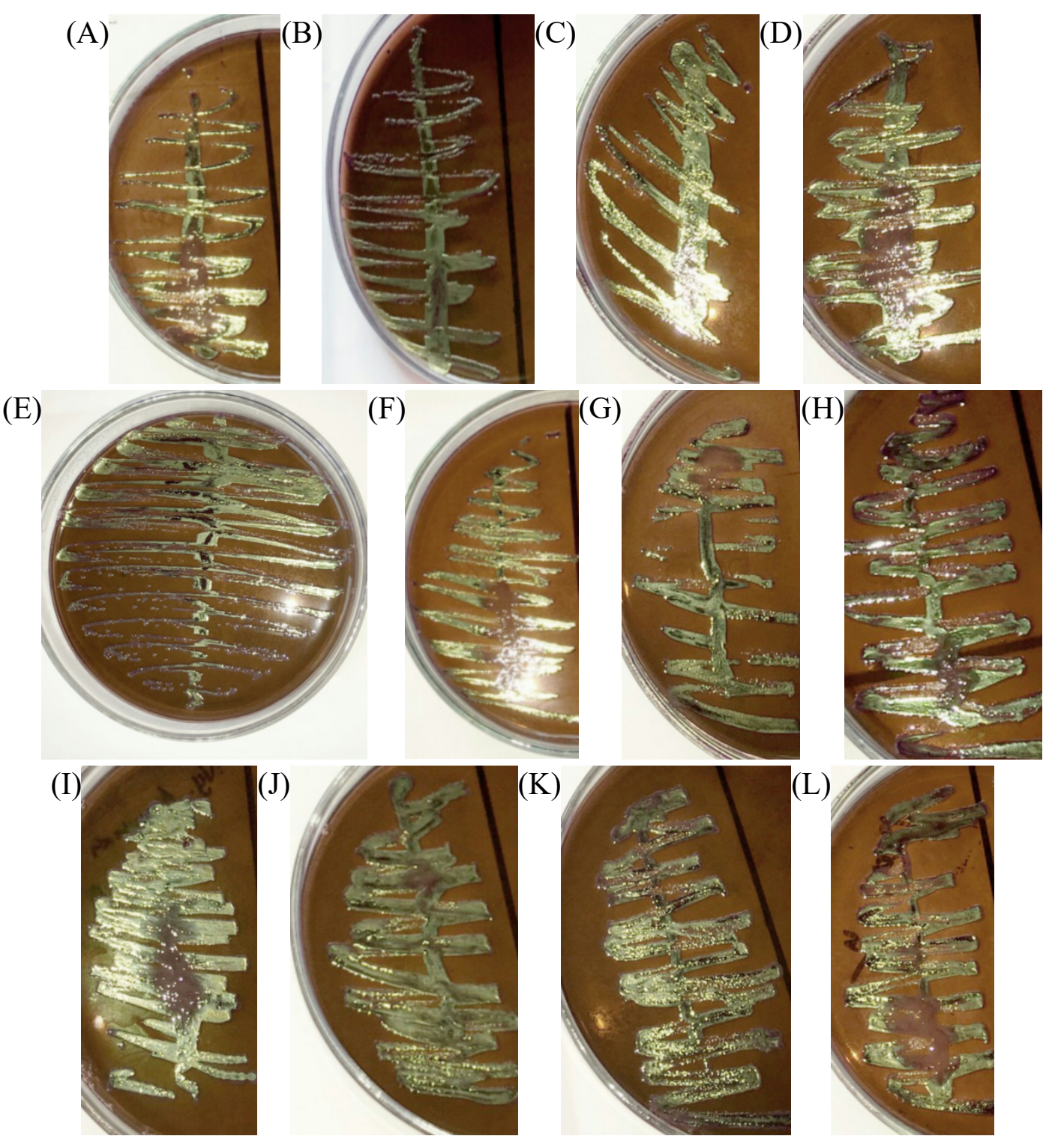

Figure 4. The Results of High Contamination Level in Diagnostic Center with Alphabetical Order A-L: 1 Second, 2 Seconds, 3 Seconds, 4 Seconds, 5 Seconds, 10 Seconds, 30 Seconds, 60 Seconds, 120 Seconds, 180 Seconds, 240 Seconds, and 300 Seconds or 5 Minutes.

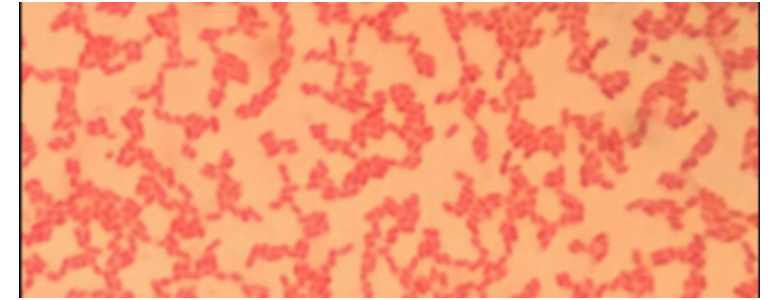

Figure 5. The Gram-Negative Microbes

At the student center's canteen 3 of 5 samples under 5 seconds are positive and 5 of 7 samples until 300 seconds are positive. The level of contamination were inconsistent (Table 1), where it could

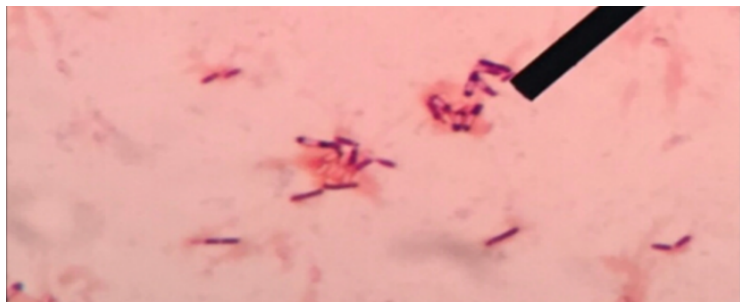

Figure 6. Positive Central-Spore (A Sign of GramPositive Microbes)

increased or decreased with increasing time, highest level of contamination on EMB to obtained in the "60 seconds" sample. Two different types of colonies can be observed in the "30 seconds" sample. 
With gram staining, gram-negative bacilli mostly founded (Figure 5), the "240 Seconds" sample showed gram-positive bacilli by central spores existence (Figure $6)$.

In the diagnostic center's canteen 12 of 12 samples were all positives (Table 2). All the samples showed good growth of immeasurable colonies with metallic green sheen indicating vigorous fermentation of lactose and acid production which precipitates the green metallic pigment observed in all EMB samples, indicating highest level of contamination since the first second (Figure 4). Gram-negative Bacilli mostly formed with gram staining (Figure 5).

\section{DISCUSSION}

Based on the results of this research increasing food contact time to the ceramic floor does not increase the concentration of specific types of microbes that are transferred to the fallen food. In this study, the student centers' canteen floor concentrations were inconsistent, one earlier time could be in higher concentration grade than the later, it also can go in higher concentration grade again. But on the diagnostic center's canteen floor the results were consistently in the highest grade, time does not give any effects at all. At Student Center's Canteen it is positive with 2 seconds, 4 seconds and 5 seconds samples; where at diagnostic center's Canteen all the samples between 1 until 5 seconds were positive.

The effect of contact time on microbial transfer is basically questionable. Miranda et al said longer contact time does influence bacterial transfer, with Longer food contact times usually resulted in transfer of more bacteria from each surface to food, ${ }^{6}$ Jensen et al stated contact time may influence transfer but generally has little effect on microbial transfer, ${ }^{7}$ Garbett et al even stated microbes transfer was not affected by contact time ${ }^{8}$. Varieties and inconsistency of food contamination with time similar with results at Student Center's canteen.

Studies also showed microbial transfer can be observed in less than 5 seconds. ${ }^{6}$ Moore indicates significant numbers of organisms can be transferred to the food even in 1 second when contacted with contaminated surface. ${ }^{9}$ This could explain One possible reason that the results were consistently high on the diagnostic center's Canteen floor is because the floors were already very contaminated from the very start. So E.Coli is already in high concentrations that makes the transfer of microbes so easy to the cheese block. It is shown that the hygiene of the diagnostic center's canteen floor hospital was really bad, based on possible E.Coli habitat most possibly the water used to clean the floor maybe we're already contaminated, or the shoes surface of people going around there were already contaminated. It may be one of possible reasons that the nosocomial infection (maybe cause diarrhea) in the hospital was still really high. A study also also stated longer food contact times did result in microbial transfer from surface to food but only when the food was placed in contact with the surface for 8 hours of more. ${ }^{1}$ This could explain why In the later time, the "240 seconds" specimen it is even shown the gram-positive microbes that contaminated the specimen. Where it is 
shown the longer the duration, more types of microbes can contaminate the food.

Transfer of bacteria from surfaces to food appear to be most affected by the moisture of the food where increased moisture level facilitates transfer. ${ }^{6,9}$ Attachment increases due to presence of biofilms; where it is affected by complex carbohydrates, nutrients and moisture. Biofilms are microscopic layers that form when bacteria excrete exogenous compounds that allow them to attach to nearly any type of surface and allow them to survive. ${ }^{1,7}$ Surface drying would be expected to cause a reduction in bacterial populations, where it is bactericidal and might be expected to severely reduce transferability. ${ }^{7,9}$ But the effect of surface drying is also questionable where high numbers of viable microbes are still observed even after the surface has been dried. ${ }^{9}$

There are multiple factors that affect microbes transfer from the surface to ceramic floor. It is dependent on the surface, food type, contact time and inoculum matrix. ${ }^{6}$ Other study reveals that bacterial transfer is dependent on produce type, surface moisture, and drying time. ${ }^{7}$ Other studies also added friction, contact pressure, initial level and volume of inoculum as important factors. ${ }^{9}$ Tile, in this case a ceramic floor, has a high transfer rate. The same as stainless steel and higher than carpet. Topography of the surface also could play an important role in microbes transfer. ${ }^{6}$

Bacterial transfer from surfaces to food could be more dependent on bacterial levels present and surface properties on the surface, and less dependent on the characteristics of the destination' food properties. the 5-second rule is "real" in the sense that longer contact time results in more transfer, it also shows that other factors including the nature of the food and the surface are of equal or greater importance. While the causes of foodborne disease are often complex, ${ }^{7}$ different in perception of Indonesia's people and western's people possibly important, that make the people in Indonesia with their perception make themselves more vulnerable with higher concentration of microbes when they eat a food within 5 minutes.

\section{CONCLUSION}

The five-second rule is a significant oversimplification of what actually happens when bacteria transfer from a surface to food. Proper and diligent sanitation of food contact surfaces is needed to reduce crosscontamination to food, because even very short contact times result in the transfer of large numbers of bacteria. Consequently, there is a need for continuous disinfection practices to reduce contamination levels available for transfer, as the risk of transfer of contamination is constantly present.

\section{REFERENCES}

1. Dawson P, Han I, Cox M, et al (2007). Residence time and food contact time effects on transfer of Salmonella Typhimurium from tile, wood and carpet: testing the five-second rule. J Appl Microbiol, 102(4), 945-953

2. Kumar P, Clark M (2012). Kumar \& Clark's Clinical Medicine $8^{\text {th }}$ ed. Spain, Saunders Elsevier 
3. Ministry of Health (2011). Situasi diare di Indonesia. Kementrian Kesehatan RI Triwulan II 2011. Available at http://www.depkes.go.id/download.ph p? file $=$ download/pusdatin/buletin/bule tin-diare.pdf. Accessed January 12, 2020

4. Bauman $\mathrm{R}$, Machunis-Masuoka $\mathrm{E}$, Montgomery J (2015). Microbiology with diseases by body system. $4^{\text {th }}$ ed. Pearson

5. ASM microbelibrary (2013). EosinMethylene blue. American society for microbiology. Available at www.microbelibrary.org/component/ $\mathrm{r}$ esource/laboratory-test/2871-eosinmethylene-blue. Accesed November 14, 2020

6. Miranda RC, Schaffner DW (2016). Longer contact times increase crosscontamination of Enterobacter aerogenes from surfaces to food. Appl Environ Microbiol, 82(21), 6490-6496

7. Jensen DA, Friedrich LM, Harris LJ, et al (2013). Quantifying transfer rates of Salmonella and Escherichia coli O157: $\mathrm{H} 7$ between fresh-cut produce and common kitchen surfaces. J Food Prot, 76(9), 1530-1538

8. Garbett (2014). Researchers prove the five-second rule is real. Aston University Birmingham UK. Available at https://www2.aston.ac.uk/news/ releases/2014/march/five-secondfood-rule-does-exist. Accessed February 20, 2020

9. Moore CM, Sheldon BW, Jaykus LA (2003). Transfer of Salmonella and Campylobacter from stainless steel to romaine lettuce. J Food Prot, 66(12), 2231-2236 